\title{
A atuação do nutricionista na Atenção Básica à Saúde em um grande centro urbano
}

\author{
The participation of the nutritionist in Primary Health Care \\ in a large urban center
}

Ana Maria Cervato-Mancuso ${ }^{1}$

Larissa Vicente Tonacio ${ }^{1}$

Erika Rodrigues da Silva ${ }^{1}$

Viviane Laudelino Vieira ${ }^{1}$

${ }^{1}$ Departamento de Nutrição, Faculdade de Saúde Pública, Universidade de São Paulo. Av. Dr. Arnaldo 715, Cerqueira Cesar. 01246-904 São Paulo SP. cervato@usp.br

\begin{abstract}
Nutritionists are important professionals for ensuring the implementation of health promotion, treatment and rehabilitation. However, their participation in primary healthcare from a quantitative standpoint is limited. The city of São Paulo has experienced an uneven urbanization process triggering new problems of insecurity in terms of food and nutrition. This article analyzes the performance of the primary healthcare nutritionist in a large urban center. It is a quantitative study that used data from the Municipal Health Department, population data of São Paulo and a semi-structured questionnaire applied in individual interviews. All regions of the city are found to have fewer nutritionists than the recommendation of the Federal Council of Nutritionists. There are 123 nutritionists in the basic healthcare network and 51 in the Family Health Support Nuclei (FHSN) (57.3\%). Each nutritionist from the FHSN accompanies 7.1 family health strategy teams on average. The age groups corresponding to children are less frequently seen by nutritionists. Comparing the activities, the transition from a model of primary health care focused on individual care to a model that prioritizes group care was observed.
\end{abstract}

Key words Primary healthcare, Human Resources, Nutrition, Urban center
Resumo O nutricionista é um profissional importante na implementação de ações de promoção, tratamento e reabilitação da saúde. Porém, sua participação na Atenção Básica (AB) é reduzida. A cidade de São Paulo vem passando por um processo desigual de urbanização, produzindo novas situações de insegurança alimentar e nutricional. Este trabalho analisará a atuação do nutricionista na $A B$ em um grande centro urbano. Trata-se de estudo de abordagem quantitativa no qual foram utilizados dados populacionais da Secretaria Municipal de Saúde e um questionário semiestruturado aplicado em entrevistas individuais. Encontraram-se 123 nutricionistas atuando na rede Básica de Saúde e 51 em Núcleos de Apoio à Saúde da Família (NASF). Todas as regiões do município apresentaram-se com menor número de nutricionistas quando comparada à recomendação do Conselho Federal de Nutricionistas. Em 57,3\% dos NASF do município identificou-se a presença deste profissional. Cada nutricionista de NASF acompanha, em média, 7,1 equipes de saúde da família. As faixas etárias que correspondem à infância são as atendidas com menor frequência pelos nutricionistas das UBS e dos NASF. Comparando-se as atividades desenvolvidas, observase a transição de um modelo de assistência primária centrado no atendimento individual para um que prioriza o atendimento em grupo.

Palavras-chave Atenção básica, Recursos humanos, Nutrição, Centro urbano 


\section{Introdução}

A criação do Sistema Único de Saúde (SUS) tem como finalidade modificar a assistência desigual à saúde da população. Sua criação foi estratégica para implementação de ações nesta área, tornando obrigatório o atendimento público e gratuito a qualquer cidadão ${ }^{1}$.

A Atenção Básica no SUS deve garantir o acesso universal aos serviços de saúde, sendo, preferencialmente, a primeira forma de atendimento à população. Dessa maneira, dependendo de sua capacidade resolutiva, a Atenção Básica pode, por meio de suas ações, resolver grande parte dos problemas e necessidades de saúde da população ${ }^{2}$. No âmbito deste nível de atenção, a Estratégia Saúde da Família (ESF), que iniciou suas atividades em 1994, pretende desenvolver ações de promoção e proteção do indivíduo, da família e da comunidade, na unidade de saúde ${ }^{3}$.

Conhecer o perfil e o processo de formação e qualificação dos profissionais que atuam na atenção básica e especificamente na equipe de saúde da família tem sido o objetivo de vários estudos ${ }^{4-}$ ${ }^{6}$, visando o que Zanetti et al. ${ }^{7}$ definem como "o fortalecimento do trabalho multiprofissional e avançando para o desenvolvimento de ações de caráter interdisciplinar que privilegiem a família como centro de atenção". Assim, em consonância com este, outros estudos vêm sendo realizados para apontar a importância de o nutricionista integrar os recursos humanos para a atenção básica à saúde ${ }^{4,6,8}$, tendo em vista que sua ausência pode resultar em execução superficial das ações relacionadas à alimentação e à nutrição ${ }^{8}$, área que vem se destacando como fundamental para a promoção de saúde.

As ações de alimentação e nutrição no âmbito da Atenção Básica visam a ampliação da qualidade dos planos de intervenção, em especial às doenças e agravos não-transmissíveis, no crescimento e desenvolvimento na infância, na gestação e no período de amamentação, evidenciando que a promoção de práticas alimentares saudáveis constitui-se em um item importante em todas as fases da vida. Assim, socializar o conhecimento sobre os alimentos e realizar ações que promovam a segurança alimentar e nutricional torna-se essencial à população9 ${ }^{9}$.

A organização de ações nas diferentes esferas de governo (federal, estadual e municipal) tem sido um dos grandes desafios relacionados às medidas de promoção da alimentação saudável. Segundo Coutinho et al. ${ }^{10}$, "promover uma alimentação saudável implica desenvolver mecanis- mos que apoiem os sujeitos a adotar modos de vida saudáveis, revendo hábitos alimentares considerados pouco saudáveis, num contexto em que a globalização e a urbanização caracterizam-se como movimentos incessantes e contínuos, altamente dificultadores do estabelecimento de práticas alimentares saudáveis, e que impactam interesses econômicos contrários à saúde pública”. Assim, segundo estes mesmos autores, a agenda de nutrição para ações de controle e prevenção das carências nutricionais e de promoção da alimentação saudável nos serviços de saúde deve ser planejada a partir do diagnóstico local, baseado na caracterização do perfil epidemiológico da comunidade e dos espaços domiciliares, sendo a atenção básica o cenário ideal para estas atividades.

Com o objetivo de ampliar o escopo das ações de Atenção Básica, a partir de 2008 foram criados os Núcleos de Apoio à Saúde da Família (NASF), que se caracterizam pela constituição em equipes multiprofissionais, pertencentes à ESF, atuantes no âmbito da saúde e assistência social, sendo o nutricionista um dos profissionais que pode fazer parte delas ${ }^{9}$. As unidades ESF contam com Equipes de Saúde da Família conforme as normas do Ministério da Saúde e obedecem as condições da área de abrangência. Já as Unidades Básicas de Saúde (UBS) possuem equipes com médicos de várias especialidades, enfermeiros, dentistas, auxiliares de enfermagem e pessoal de apoio técnico. A demanda atendida apresenta-se como espontânea e/ou encaminhada por outros serviços, sendo que a delimitação da área de abrangência se refere exclusivamente às ações de vigilância à saúde ${ }^{5}$. A ESF reorganiza a prática assistencial a partir da atenção básica, em substituição ao modelo assistencialista característico, direcionado à cura de doenças e agravos. Possui dinâmica orientada para a promoção da qualidade de vida, permitindo identificação mais acurada e melhor acompanhamento de doenças crônicas ${ }^{11}$.

No que se refere aos grandes centros urbanos, Pádua e $\mathrm{Boog}^{6}$, por sua vez, elucidam as dificuldades relativas à promoção da segurança alimentar e nutricional nestas cidades. Estes autores identificam a inserção do nutricionista na Rede Básica de Saúde na região de Campinas, mas observam que o número elevado de habitantes é um obstáculo aos serviços de saúde, uma vez que estes não são suficientes para atender a população devidamente.

Tomando como base as considerações expostas, o pressuposto aqui estabelecido é que uma adequada inserção profissional favorece a pro- 
moção da saúde a partir da qualificação das ações de alimentação e nutrição tendo como foco a saúde da família. Neste sentido, a premissa desta pesquisa está relacionada ao fato de o nutricionista ser um componente da equipe de saúde e o grau de sua inserção na assistência à saúde da população tem aumentado em função da implantação dos NASF nas diferentes regiões do país e que desta forma quanto maior essa adequação melhor será o resultado da prestação de serviços de saúde à população.

Considerando que a cidade de São Paulo é uma das mais populosas do mundo, que vem passando por novas situações de insegurança alimentar e nutricional e que a inserção do nutricionista na atenção básica é um processo recente, o presente artigo tem como objetivo analisar a atuação do nutricionista da Atenção Básica em um grande centro urbano.

\section{Métodos}

Trata-se de um estudo de abordagem quantitativa que utilizou dados disponíveis sobre nutricionistas contratados pela Secretaria Municipal de Saúde (SMS/SP), dados populacionais do município de São Paulo e, como instrumento de coleta de informação, um questionário semiestruturado aplicado em entrevistas individuais. Pela proposta de análise do município como uma unidade, trata-se também de um estudo de caso.

O trabalho foi realizado no período de março a novembro de 2010, no município de São Paulo. As seguintes variáveis foram analisadas: quantidade de nutricionistas, quantidade de habitantes por coordenadoria regional de saúde, quantidade de ESF (dos NASF com nutricionistas), vulnerabilidade social do local atendido pelas coordenadorias e atividades desenvolvidas pelos nutricionistas.

Para o cálculo e a análise dos dados, foram consideradas como regiões as coordenadorias regionais de saúde estabelecidas pela SMS/SP, sendo, portanto, divididas em: Centro-oeste, Leste, Norte, Sudeste e Sul.

Os dados sobre a quantidade de nutricionistas foram obtidos por meio da Secretaria Municipal de Saúde; e os dados populacionais, pelo censo demográfico do Instituto Brasileiro de Geografia e Estatística (IBGE) ${ }^{12}$. A partir destes dados, foi calculada a porcentagem de adequação da quantidade de nutricionistas por dois critérios de referencia: um do Conselho Federal de Nutricionistas $(\mathrm{CFN})^{13} \mathrm{e}$ outro do Ministério da Saúde ${ }^{14}$.
Como referência para o cálculo da porcentagem de adequação de nutricionistas da Atenção Básica, utilizou-se a recomendação da resolução do CFN no 380/2005 $5^{13}$, a qual preconiza um nutricionista para cada 50 mil habitantes. A partir desta recomendação, calculou-se a quantidade de nutricionistas recomendada para cada região de São Paulo, tendo em vista o número de habitantes indicado pelo censo do IBGE. Em seguida, com base nos dados populacionais encontrados, comparou-se a quantidade de nutricionistas existentes em cada região com a recomendação, calculando-se a porcentagem de adequação.

Quanto aos nutricionistas dos NASF, foi estabelecida a relação entre a quantidade de equipes da ESF e habitantes por nutricionista do seguinte modo: uma ESF a cada 4.000 habitantes, estabelecido pela portaria $648 / 06^{14} \mathrm{e}$, considerando o número máximo de 20 ESF por NASF com nutricionista, um nutricionista atenderia $80 \mathrm{mil}$ habitantes. O número de equipes da ESF por NASF foi obtido por meio do Cadastro Nacional de Estabelecimentos de Saúde (CNES) ${ }^{15}$.

A fim de estabelecer relação entre a vulnerabilidade social da população e a localização e cobertura das equipes, utilizou-se o Índice Paulista de Vulnerabilidade Social (IPVS) segundo setor censitário de 2.000 da Fundação Seade ${ }^{16}$. Para avaliar as regiões foram classificadas as faixas de vulnerabilidade social em: (1) regiões que não possuem vulnerabilidade social ou, quando possuem, são classificadas como grau muito leve ou leve; (2) regiões que no centro não possuem vulnerabilidade social ou, quando possuem, variam de muito leve a leve, enquanto que na periferia, são encontrados índices de muito alta, alta e média vulnerabilidade social; e (3) regiões que possuem média, alta ou muito alta vulnerabilidade social.

$\mathrm{Na}$ terceira etapa, verificaram-se as atividades desenvolvidas pelos nutricionistas alocados nas equipes das UBS e dos NASF por meio de entrevistas individuais. Solicitou-se que o profissional enumerasse as quatro atividades que desenvolvia com maior frequência, bem como a carga horária semanal aproximada despendida para cada uma delas. Além disso, questionou-se quanto às faixas etárias com as quais atuam mais frequentemente.

A partir dos relatos, foram calculadas as porcentagens correspondentes a cada atividade e das faixas etárias que eram atendidas com maior frequência por estes profissionais.

A pesquisa foi aceita pelos Comitês de Ética em Pesquisa da Faculdade de Saúde Pública da 
Universidade de São Paulo e da Secretaria Municipal da Saúde de São Paulo.

\section{Resultado}

Encontraram-se 123 nutricionistas atuando na Rede Básica de Saúde. A Tabela 1 mostra a distribuição destes nutricionistas por regiões do município de São Paulo.

As regiões Leste e Sul apresentam, respectivamente, $50 \%$ e $31 \%$ da quantidade de nutricionistas recomendada. A região Norte, que apresenta um grau de vulnerabilidade média, é a segunda em inadequação (37\%). O grau de adequação da inserção profissional das regiões Centro-oeste e Sudeste mostra-se superior, quase alcançando o recomendado com, respectivamente, $96 \%$ e $88 \%$ de adequação. Tais regiões mostram-se praticamente sem vulnerabilidade social.

Por meio dos dados expostos na Tabela 1, é possível verificar que todas as regiões do município de São Paulo apresentam-se com quantidade menor de nutricionistas quando comparada à quantidade recomendada pela resolução, sendo que as regiões socialmente mais vulneráveis são aquelas com menor número de nutricionistas.
Com relação à quantidade de nutricionistas atuantes nos NASF verificou-se a participação do profissional em 51 das 89 equipes existentes no município, representando $57,3 \%$ dos NASF com nutricionista. A Tabela 2 mostra a distribuição dos NASF com nutricionistas pelas regiões do município.

O grau de inserção do nutricionista nas Equipes de NASF é desigual entre as regiões. Cada um de NASF acompanha, em média, 7,1 equipes de saúde da família. Apenas na região Norte, este valor alcança a faixa estabelecida pela lei ( 8 a 20 equipes de Saúde da Família). Considerando que cada nutricionista do NASF atenda, direta ou indiretamente, 80 mil habitantes, verifica-se que seriam necessários 133 nutricionistas para atender a população total do município de São Paulo.

Observa-se que as regiões mais vulneráveis (Leste e Sul) apresentam maior número de nutricionistas em NASF em comparação com as regiões menos vulneráveis (centro-oeste e sudeste).

Referente ao atendimento nutricional por faixas etárias dos nutricionistas das UBS, verificase que as atendidas com maior frequência corresponde à idade adulta $(19,1 \%)$ como elucida o Gráfico 1. As faixas que agrupam as crianças menores de seis meses $(10,6 \%)$ e as entre seis

Tabela 1. Comparação entre a quantidade real de nutricionistas e a recomendada pela legislação para as regiões do município, São Paulo 2010.

\begin{tabular}{lrccc}
\hline \multicolumn{1}{c}{ Região } & $\begin{array}{c}\text { Número de } \\
\text { habitantes }\end{array}$ & $\begin{array}{c}\text { Grau de } \\
\text { vulnerabilidade social }\end{array}$ & $\begin{array}{c}\text { Número de } \\
\text { nutricionistas }\end{array}$ & $\begin{array}{c}\text { Porcentagem de adequação } \\
\text { recomendada }\left(^{*}\right)\end{array}$ \\
\hline Centro-oeste & 1244456 & 1 & 24 & 96 \\
Sudeste & 2499294 & 1 & 44 & 88 \\
Leste & 2396940 & 2 & 24 & 50 \\
Norte & 2136977 & 2 & 16 & 37 \\
Sul & 2402093 & 3 & 15 & 31 \\
Total & $\mathbf{1 0 . 6 7 9 . 7 6 0}$ & & $\mathbf{1 2 3}$ & $\mathbf{5 7 , 5}$ \\
\hline
\end{tabular}

$\left.{ }^{*}\right)$ quantidade existente/quantidade recomendada x 100 .

Tabela 2. Quantidade de nutricionistas atuantes em NASF por região do município de São Paulo, equipes de saúde da família com nutricionistas e número médio de equipes orientadas por nutricionista por região do município, São Paulo, 2010.

\begin{tabular}{lcccc}
\hline \multicolumn{1}{c}{ Região } & Nutricionistas $\left(\mathbf{n}^{\mathbf{o}}\right)$ & Porcentagem $(\%)$ & ESF $\left(\mathbf{n}^{\mathbf{o}}\right)$ & Equipes por nutricionista \\
\hline Centro-oeste & 6 & 12 & 37 & 6,2 \\
Sudeste & 1 & 2 & 6 & 6,0 \\
Leste & 15 & 29 & 85 & 5,7 \\
Norte & 7 & 14 & 70 & 10,0 \\
Sul & 22 & 43 & 166 & 7,5 \\
Total & $\mathbf{5 1}$ & $\mathbf{1 0 0}$ & $\mathbf{3 6 4}$ & $\mathbf{7 , 1}$
\end{tabular}


meses e dois anos $(11,3 \%)$ têm a menor frequência de atendimento direto pelos profissionais.

No que se refere ao nutricionista do NASF, verifica-se que o padrão de atendimento é semelhante quando comparado aos que atuam na UBS. No Gráfico 1 é possível observar que as crianças menores de 6 meses são as atendidas com menor frequência, constando em $13 \%$ das respostas das nutricionistas e, em seguida, as crianças entre 6 meses e 2 anos de idade (13,7\% das respostas). A faixa etária que se mostrou mais citada foi a que compreende a idade adulta, entre 20 e 60 anos de idade (18,5\%).
Quanto às atividades desenvolvidas pelos nutricionistas das UBS, há predomínio dos atendimentos individuais $(24,7$ horas por semana, correspondente a $61,5 \%$ da carga horária). Além desta atividade principal e atividades em grupo, realizam outras como: atividades administrativas, visitas domiciliares, elaboração de dietas e reuniões (Gráfico 2).

Dentre as atividades desenvolvidas pelos nutricionistas dos NASF, destacam-se os grupos e, em seguida, aparecem as reuniões. As visitas domiciliares, o atendimento individual, o matriciamento (apoio matricial), o atendimento com-

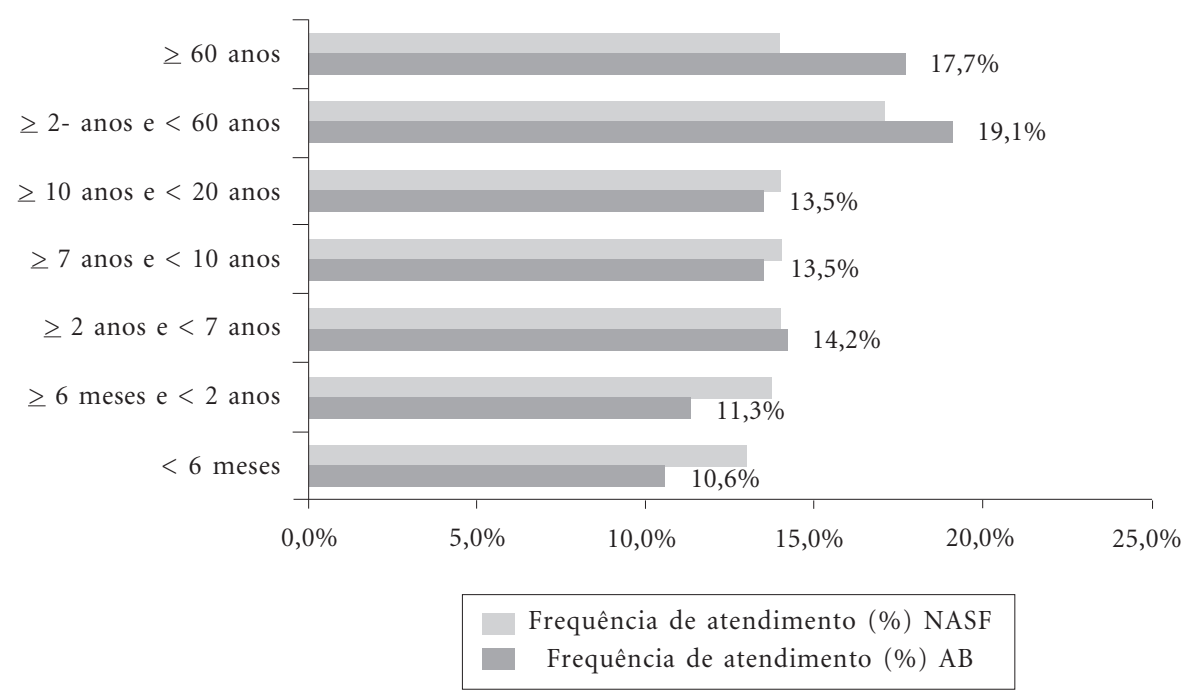

Gráfico 1. Frequência da citação do atendimento realizado pelos nutricionistas dos NASF e das UBS de acordo com as faixas etárias, São Paulo, 2010.

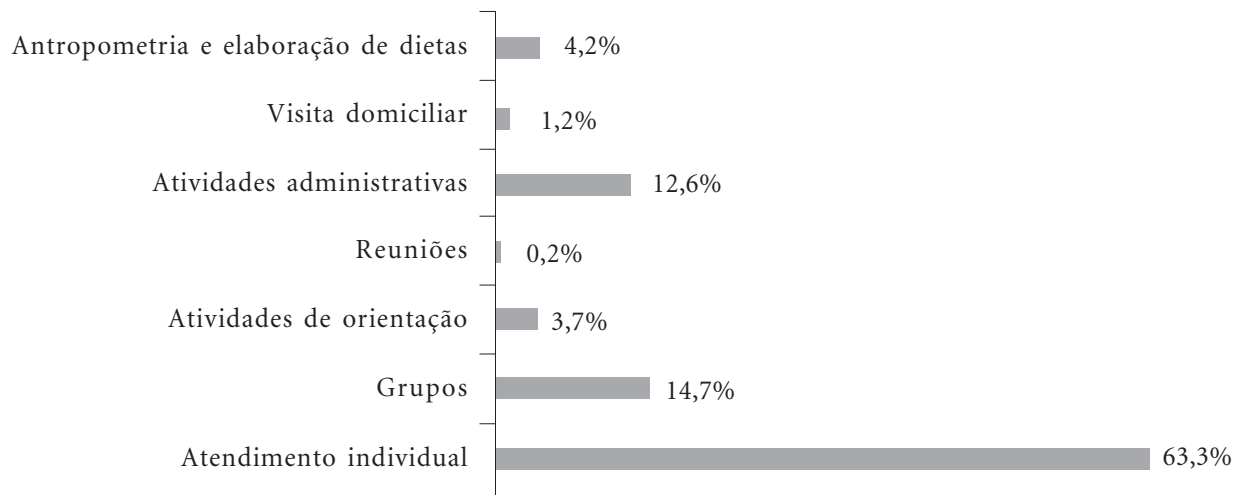

Gráfico 2. Relação das atividades desenvolvidas pelos nutricionistas das UBS entrevistados e suas respectivas frequências, São Paulo, 2010. 
partilhado e as atividades administrativas foram as outras atividades citadas (Gráfico 3).

\section{Discussão}

A atuação do nutricionista no município de São Paulo ocorre em diferentes contextos sociais e suas principais atividades de prestação de serviços diretos à população como atendimentos individuais e atendimento em grupo são desenvolvidos tanto na UBS quanto no NASF. Entretanto, sua forma da inserção e atuação nestas duas áreas tem aspectos convergentes e divergentes.

Comparando-se os dados, verifica-se predominância dos nutricionistas do NASF nas regiões do município com maior vulnerabilidade social (Sul e Leste), enquanto que há maior número destes profissionais nos estabelecimentos de atenção básica das regiões Centro-oeste e Sudeste, caracterizados pelo menor IPVS. Essa presença em contextos diferentes pressupõe necessidades de saúde da população diferentes e caracteriza a implantação dos NASF com nutricionistas prioritariamente nas regiões do município mais vulneráveis.

De acordo com o estudo desenvolvido por Bousquat et al. ${ }^{17}$, a ESF, intitulada na época Programa Saúde da Família (PSF), foi implantada primordialmente nas regiões periféricas e de alta exclusão social, cujas famílias possuíam renda de até cinco salários mínimos e segundo Henrique e Calvo ${ }^{3}$, em áreas de risco nutricional. Após esse período inicial, expandiu-se rapidamente e, em janeiro de 2001, já existiam equipes nos 27 estados da Federação, atingindo cerca de 90\% dos municípios brasileiros.

Em nível nacional o processo de implantação do PSF, predominou em municípios de pequeno porte, nos quais uma equipe respondia por elevada cobertura populacional. Por outro lado, na grande maioria das cidades de médio e grande porte, a ESF persiste com as características de uma estratégia de expansão dos cuidados básicos de saúde, voltada para grupos populacionais sob maior risco social e expostos a precárias condições sanitárias e não como estratégia substitutiva ${ }^{3,10}$.

No município de São Paulo, a inserção do nutricionista acompanhou o modelo de implementação da Estratégia Saúde da Família no país dado a sua presença na rede básica de saúde ser mais frequente em áreas de menor vulnerabilidade social enquanto no NASF a inserção ocorre em regiões mais vulneráveis.

A implementação do PSF como política de governo com suas novas diretrizes do modelo assistencial de saúde encontram várias dificuldades, dentre as quais Viana et al. ${ }^{18}$ apontam para a presença de uma assistência baseada no modelo biomédico de saúde, a demanda excessiva de usuários, a precarização do trabalho dos profissionais e a escassa capacitação em serviço que se articulam com a questão "recursos humanos". Segundo estes autores, nos grandes centros urbanos observa-se, ainda, a maior disponibilidade de oferta de serviços de saúde, particularmente os de média e alta complexidade, que se constitui também como barreira ao acesso e oferta de ações básicas. A disponibilidade destes serviços é reflexo direto do modelo assistencial curativo,

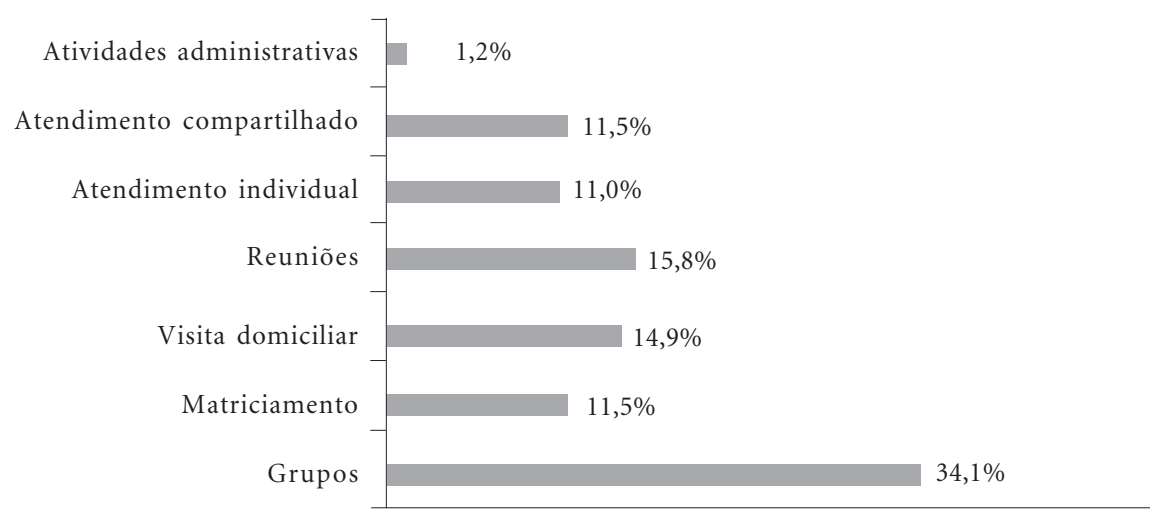

Gráfico 3. Relação das atividades desenvolvidas pelos nutricionistas dos NASF entrevistados e suas respectivas frequências, São Paulo, 2010. 
que minimiza a importância das ações básicas de saúde, quando, na verdade, todos os níveis de atenção à saúde deveriam ser resolutivos, hierárquicos e harmônicos entre si.

Como esse trabalho foi realizado no município de São Paulo, onde a ESF foi implantada em 2008 e a presença dos nutricionistas em UBS é anterior a esse processo, sua atuação em UBS pode representar um modelo de atenção tradicional, enquanto que no NASF representa o novo modelo, significando a presença dos dois modelos de assistência na área de alimentação e nutrição, convivendo no sistema local de saúde.

O nutricionista do NASF foi incorporado ao município por conta da implantação da ESF, que aconteceu após a municipalização, em 2001, dos serviços de saúde, os quais já incorporavam esse profissional. Essa municipalização, segundo Pinto et al. ${ }^{19}$, fez com que o município de São Paulo passasse a ter uma rede pública de serviços de saúde grande e bastante diversificada, com mais de 200 unidades ambulatoriais. Somado a esta mudança, atualmente aquelas unidades ambulatoriais com espaço físico e estrutura melhores foram transformadas em ambulatórios de especialidades (AE), outras incorporaram equipes da ESF e, a partir de 2005, outras incluíram um serviço de pronto atendimento denominado Assistência Médica Ambulatorial (AMA). Esse é o sistema no qual o nutricionista de UBS está inserido reforçando um cenário propício para atenção direcionada ao indivíduo.

O resultado da presente pesquisa aponta que a inserção do nutricionista nos NASF é considerável: mais da metade dos núcleos possuem este profissional na equipe. Há, entretanto, desigualdade na sua distribuição geográfica dada sua maior presença em regiões de alta vulnerabilidade social. Tal fator possibilita a exclusão do atendimento de populações vulneráveis em regiões consideradas de vulnerabilidade média ou baixa como, por exemplo, a região Norte, que apresenta na maior parte do território média vulnerabilidade social e, ao mesmo tempo, algumas áreas de altíssima vulnerabilidade.

Considerando o grau de vulnerabilidade social em regiões como a Leste e Sul e o perfil de desigualdade socioeconômica de São Paulo, a demanda populacional para tratamento de agravos nutricionais é um grande desafio para viabilizar um modelo de assistência que atenda, cure, oriente e promova coparticipação dos usuários com base em práticas promotoras de saúde ${ }^{10}$. Agregado a isso, o desafio se amplia quando se compara a situação encontrada e a recomenda- da, verificando-se déficit numérico de nutricionistas nas duas áreas.

Os parâmetros numéricos propostos pelo CFN por área de atuação foram os primeiros a nortear a adequação da presença destes profissionais, não existindo outras referências para a área. Alguns estudos realizados antes da criação dos referidos parâmetros, como o de Gambardella et $a l .{ }^{20}$, que iniciaram esta discussão sobre a avaliação quantitativa da inserção profissional e outros, como o de Pádua e Boog ${ }^{21}$ e o de Santos e Diez-Garcia' ${ }^{22}$, que analisaram a inserção numérica do nutricionista nas áreas da saúde pública e clinica, respectivamente. Assim como o resultado da presente pesquisa, estas outras apontam para inadequada inserção deste profissional em diferentes áreas da saúde. Entretanto, é possível perspectivar uma mudança no grau desta inserção na área da atenção básica o que possibilitaria a ampliação de suas atividades.

Assis et al. ${ }^{4}$ discutem sobre a necessidade do nutricionista no PSF como peça fundamental para avançar as condições técnicas e operacionais em direção à garantia da segurança alimentar e nutricional. Segundo a autora:

a competência do nutricionista para integrar a equipe do PSF está estabelecida em sua formação acadêmica, a qual o instrumentaliza a realizar $o$ diagnóstico nutricional da população, tornando-o, assim, o único profissional a receber uma instrução especifica que lhe permite, a partir desse diagnóstico e da observação dos valores socioculturais, propor orientações dietéticas cabíveis e necessárias, adequando-as aos hábitos da unidade familiar, à cultura, às condições fisiológicas dos grupos e à disponibilidade de alimentos. Trata-se, portanto, de um profissional apto a participar efetivamente da recriação das práticas de atenção à saúde no Brasil.

A Portaria $154 / 08^{9}$, que implementa o NASF, dispõe sobre a composição dos núcleos e deixa a cargo dos gestores municipais, a escolha dos profissionais que irão compor a equipe seguindo critérios identificados a partir das necessidades locais e da disponibilidade de profissionais.

No que se refere às necessidades da população paulistana, estudo realizado na região oeste, especificamente no Jardim Jaqueline, o mais crítico bolsão de pobreza da região, verificou altíssimo índice de privação e vulnerabilidade social agregado à identificação de $88,0 \%$ das famílias vivendo em situação de insegurança alimentar ${ }^{23}$. A insegurança alimentar está relacionada a problemas de acesso à alimentação adequada e associada com distintos distúrbios nutricionais, dentre eles a desnutrição ${ }^{24}$. A presença do profissio- 
nal nestas regiões torna-se fundamental para integrar a equipe com seus conhecimentos sobre alimentação e nutrição e assim, como refere Mattos e Neves ${ }^{25}$, reafirmar o modelo de atenção proposto pelo país dado que é o profissional com conhecimento específico que pode propor as orientações dietéticas adequadas à realidade de cada unidade familiar.

Os dados mostram que, em geral, os núcleos com nutricionistas na equipe acompanham menos ESF do que o recomendado. Nesse sentido, o resultado não corresponde ao critério estabelecido, o que traz uma reflexão sobre as condições que as equipes enfrentam para o cumprimento de suas atividades. Uma vez que o NASF vem com o propósito de trabalho em conjunto, tanto entre as equipes multiprofissionais do NASF quanto entre a ESF e os profissionais da UBS, o planejamento das ações conjuntas entre os profissionais da ESF e da UBS pode não acontecer. Assim, não há interação suficiente para a criação de ações e estratégias que englobem o ser humano em sua totalidade, o que contribui para a continuação do modelo de saúde que fragmenta o atendimento e o realiza por meio do binômio queixa-conduta.

A maior parte dos NASF com nutricionistas está localizado nas regiões periféricas e mais vulneráveis, que apresentam condições socioeconômicas baixas. Isso exige do nutricionista o desenvolvimento de competências para compreensão da realidade local, assim como para o planejamento das etapas e ações intersetoriais necessárias, a fim de garantir os direitos assegurados. O direito humano a alimentação adequada, moradia digna, educação e saúde devem ser buscados pelo profissional para garantir a segurança alimentar e nutricional dessa população. Para garantir estes e outros direitos relacionados à segurança alimentar e nutricional, as Diretrizes Curriculares dos Cursos de Nutrição ${ }^{26}$ apontam o nutricionista como o profissional da área da saúde capacitado para trabalhar com questões de insegurança alimentar e nutricional dos diferentes grupos populacionais.

Ao analisar a prática profissional, em relação ao atendimento nutricional por faixas etárias, nota-se maior homogeneidade no NASF em comparação à UBS, correspondendo à proposta da ESF, que visa o atendimento das necessidades da família como um todo.

Tanto os nutricionistas dos NASF quanto os das UBS relataram atender com menor frequência as crianças de 0 a 2 anos. É importante salientar que, se o NASF foi propositalmente inserido nas regiões periféricas e de maior vulnerabilidade social e que um dos critérios adotados para a classificação das regiões em graus de vulnerabilidade social é a presença de crianças pequenas (de 0 a 4 anos) nas famílias, era de se esperar a citação desta faixa etária de forma mais preponderante. Tais dados vão ao encontro das estatísticas da população infantil, a qual se mostra cada vez mais atingida pela insegurança alimentar e nutricional. Segundo Fernandes et al. ${ }^{27}$, as crianças menores de 10 anos de idade são o segundo grupo etário mais atendido pela ESF, perdendo somente para o grupo de adultos de 20 a 39 anos. Verifica-se, pois, que essa população está sendo atendida pela equipe, mas não é assistida diretamente pelo nutricionista do NASF.

Comparando-se as atividades dos nutricionistas do NASF com os das UBS, é possível distinguir claramente a mudança de um modelo de assistência individualista e biologicista, centrado no binômio queixa-conduta e que prioriza o atendimento individual, para um modelo que trata do indivíduo em sua integridade, visando à atuação de uma equipe multidisciplinar que reúne conhecimentos de todas as áreas que o compõe, focando o atendimento em grupo. Visa também à prevenção de agravos e promoção da saúde, em detrimento do modelo antigo de assistência, que prioriza a cura ou a amenização dos sintomas das doenças e agravos.

Elias et al. ${ }^{5}$ analisaram a opinião dos usuários em relação ao modelo de assistência do PSF e o modelo tradicional de atenção básica. A opinião dos usuários em relação ao modelo de assistência do PSF é comparativamente melhor do que a do modelo tradicional de atenção básica em todos os estratos de exclusão social do município de São Paulo, sendo que pode ser estabelecida uma relação inversa entre o grau de aprovação do serviço e a exclusão dos estratos, isto é, quanto maior a exclusão, menor o índice de aprovação ao PSF. Provavelmente, segundo estes autores, se por um lado os estratos com menor exclusão têm acesso a uma maior densidade de equipamentos de saúde, permitindo-lhes o atendimento de suas necessidades; por outro, a capacidade do PSF em resolver as demandas dos usuários não é a mesma do modelo tradicional das UBS, que geralmente conta com médicos especializados.

É importante destacar que a resolução 380 do CFN, que estabelece os parâmetros numéricos de referência por área, dispõe sobre as áreas de atuação e as respectivas atribuições. Assim, estão descritas as competências do nutricionista no exercício das atribuições na área da saúde co- 
letiva, o que orienta e sustenta as ações deste profissional na atenção básica que inclui as atividades de promoção e de assistência à saúde.

O estudo realizado por $\mathrm{Maia}^{28}$ com o objetivo de identificar as práticas de trabalho exercidas pelo nutricionista na atenção primária da $\mathrm{Mi}$ crorregião de Saúde de Viçosa, MG, verificou como atribuição a supervisão de estágios dos cursos de nutrição, as visitas domiciliares e as atividades de assistência a saúde por demanda espontânea ou referenciada. Verifica-se, assim, a diversidade de ações realizadas pelo profissional.

As matrizes de ações em alimentação e nutrição estabelecidas pelo Ministério da Saúde relacionam e classificam as ações pertinentes ao profissional de nutrição em ações de diagnóstico, promoção da saúde, prevenção de doenças e assistência, tratamento e cuidado ${ }^{29}$. Nas atividades desenvolvidas pelos nutricionistas que atuam em UBS, há o predomínio de frequência de ações classificadas na matriz como de assistência, tratamento e cuidado (atendimento individual, prescrição dietética e visita domiciliar), com grupos educativos sendo caracterizados como atividade de promoção da saúde. Por outro lado, os nutricionistas que atuam nos NASF tiveram predominância em atividades de promoção da saúde (grupos educativos), acompanhado de ações de prevenção de doenças (atendimento compartilhado), bem como atividades que tem como foco o planejamento de ações nesta área (reuniões e matriciamento), com pouca frequência em ações de assistência (atendimento individual) e atividades administrativas.

Durante a análise das atividades evidenciouse uma limitação do estudo: a falta de padronização de suas nomenclaturas. Um exemplo é a citação da atividade "matriciamento". Para Campos e Domitti ${ }^{30}$ o apoio matricial como modo de trabalho pode ser entendido como uma metodologia que pressupõe construções compartilhadas entre a equipe de referência e os apoiadores, que vem com a missão de agregar conhecimentos à equipe de referência e auxiliar na resolução de casos. Assim, seria um "jeito de fazer" e não uma atividade propriamente dita. Outro exemplo está presente nas entrevistas com os nutricionistas das UBS, onde foi relatada a elaboração de dietas, quando isto poderia ser entendido como um procedimento dentro de uma determinada atividade.

Comparando tais dados é possível verificar, mais uma vez, a presença simultânea do modelo tradicional de atendimento, que busca suprir a demanda de agravos à saúde já instaurados e com foco no atendimento individual; e o modelo baseado na promoção da saúde e na prevenção de agravos, que prioriza o atendimento em grupo.

\section{Considerações finais}

A inserção profissional do nutricionista na atenção básica em grandes centros urbanos é uma atividade que vem se ampliando principalmente com a implantação dos Núcleos de Apoio à Saúde da Família. Esta pesquisa procurou analisar a inserção do nutricionista na atenção básica no município de São Paulo.

Os profissionais estudados estavam alocados em duas áreas de assistência à saúde: unidade básica e como integrante do NASF. As UBS com nutricionistas estão localizadas fundamentalmente em regiões de menor vulnerabilidade social enquanto os NASF que tem este profissional integrado à equipe estão em regiões de maior vulnerabilidade. Assim, as regiões sudeste e centro-oeste contam com nutricionistas em UBS, enquanto as leste e sul em NASF. A ampliação da inserção profissional no município por meio da inclusão de um novo modelo de atenção baseado na ESF acontece em regiões onde há menor numero de nutricionistas nos estabelecimentos de saúde, especificamente, em UBS.

Também foi verificado que o grau de inserção deste profissional é insuficiente tomando por base os parâmetros adotados pela presente pesquisa. Os nutricionistas tanto em UBS quanto nos NASF tem como desafio a atenção a uma população numerosa e muitas das equipes destes níveis de assistência não contam com este profissional. Neste caso, as ações de alimentação e nutrição desenvolvidas por estas equipes podem estar inadequadas ou, no mínimo, insuficientes diante das necessidades da população e condicionadas à definição de prioridades pelo profissional ou pela própria equipe.

Para a análise da atuação dos profissionais, optou-se pela identificação das principais atividades e da faixa etária mais frequentemente atendida. Apesar das limitações do estudo como a falta de padronização da nomenclatura dos procedimentos e das ações citadas, esse estudo permitiu verificar que a atuação nestas duas áreas apresenta similaridades no que se refere ao tipo de atividade e à população atendida. Entretanto, a definição de prioridade na atenção pode ser diferente dado que a atenção individual recebe destaque na atuação do nutricionista em UBS enquanto que como integrante dos NASF, as ati- 
vidades em equipe ou com grupos populacionais são mais citadas. O profissional alocado em UBS tem suas atividades mais frequentemente relacionadas ao atendimento individual e, considerando a faixa etária, destinados aos adultos e idosos. É possível inferir que estas ações estão focadas no tratamento dietético de indivíduos portadores de doenças e agravos não transmissíveis.

Conforme exposto, a inserção do nutricionista contribui no processo atenção à saúde da população por meio das atividades de promoção e de assistência e isso, em certo grau, está acontecendo no município de São Paulo. Esse resultado se refere aos serviços municipais, o que é um ponto positivo, na medida em que denota uma postura pró-ativa frente às novas exigências de um modelo de atenção integral.

Os dados analisados mostram que os nutricionistas dos NASF encontram-se em maior quantidade exatamente nas regiões onde falta o nutricionista que prioriza o atendimento individual e vice-versa. Será possível que as ações destes profissionais estejam se complementando? Neste caso, quais ações estarão sendo realizadas: promoção à saúde, prevenção e/ou tratamento?

Foi possível verificar que o nutricionista atuante no município de São Paulo, tanto na UBS quanto no NASF, tem pela frente o grande desafio de integração de atividades da área de alimentação e nutrição, considerando que estão preferencialmente em regiões distintas, possibilitando ações não complementares. Além disso, suas ações nestas duas áreas estão pautadas diretamente para o indivíduo ou grupos populacionais com pouca menção a ações intersetoriais.

O município de São Paulo como um grande de centro urbano com regiões de alta vulnerabilidade social e problemas nutricionais, mesmo com esses avanços verificados, ainda não possui um número adequado de nutricionistas, sendo este um dos pontos importantes e que necessitam de outros tipos de avaliações dado a existência de outros serviços presente no município. Pode-se concluir que as ações realizadas pelos profissionais estão incompletas, pela quantidade insuficiente de NASF com nutricionista e pela quantidade de equipes apoiadas por esses núcleos e pelo conflito da existência de dois modelos de assistência,

\section{Colaboradores}

AM Cervato-Mancuso, LV Tonacio, ER Silva e VL Vieira participaram igualmente em todas as etapas da elaboração do artigo 


\section{Referências}

1. Brasil. SUS de A a $Z$ [página da Internet]. Brasil: Ministério da Saúde. [acessado em 2011 jan 07]. Disponível em: http://portal.saude.gov.br/portal/ saude/cidadao/default.cfm.

2. São Paulo. Política de apoio à atenção básica no SUS/ SP [documento da Internet]. São Paulo: Secretaria Municipal da Saúde [acessado em 2011 jan 07]. Disponível em: http://www.saude.sp.gov.br/resources/gestor/projetos/politica_de_apoio_a_atencao_ basica_no_estado_de_sp.pdf.

3. Henrique F, Calvo MCM. Grau de implantação do Programa Saúde da Família e indicadores sociais. Cien Saude Colet 2009; 4(Supl. 1):1359-1365.

4. Assis AMO, Santos SMC, Freitas MCS, Santos JM, Silva MCM. O Programa Saúde da Família: contribuições para uma reflexão sobre a inserção do nutricionista na equipe multidisciplinar. Rev Nutr 2002; 15(3):255-266.

5. Elias PE, Ferreira CW, Alves MCG, Cohn A, Kishima V, Escrivão Junior A, Gomes A, Bousquat A. Atenção Básica em Saúde: comparação entre PSF e UBS por estrato de exclusão social no município de São Paulo. Cien Saude Colet 2006; 11(3):633-641.

6. Pádua JG, Boog MCF. Avaliação da inserção do nutricionista na Rede Básica de Saúde dos municípios da Região Metropolitana de Campinas. Rev Nutr 2006; 19(4):413-424.

7. Zanetti TG, Perlini NMOG, Van der Sand ICP. Equipes de Saúde da Família de uma coordenadoria regional de saúde do Rio Grande do Sul: perfil socioprofissional e formação dos profissionais. Rev Enferm UFPE On line [periódico na Internet]. 2010 Out-Dez [acessado 2011 jan 15]; 4(4):[cerca de 6 p.]. Disponível em: http://www.ufpe.br/revista enfermagem/index.php/revista/article/view/1084/ pdf_219

8. Santos AC. A inserção do nutricionista na Estratégia de Saúde da Família: o olhar de diferentes trabalhadores da saúde. Fam Saúde e Desenvol 2005; 7(3):257-265

9. Brasil. Portaria no 154 de 24 de janeiro de 2008. Cria os Núcleos de Apoio à Saúde da Família (NASF). Diário Oficial da União 2008; 24 jan.

10. Coutinho JG, Gentil PC, Toral N. A desnutrição e a obesidade no Brasil: o enfrentamento com base na agenda única de nutrição. Cad Saude Publica 2008; 24(Supl. 2):S332-S340.

11. Paiva DCP, Bersusa A AS, Escuder MML. Avaliação da assistência ao paciente com diabetes e/ou hipertensão pelo Programa Saúde da Família do $\mathrm{Mu}$ nicípio de Francisco Morato, São Paulo, Brasil. Cad Saude Publica 2006; 22(2):377-385.

12. São Paulo. População recenseada, projetada, taxas de crescimento populacional e densidade demográfica do município de São Paulo, subprefeituras e distritos municipais 1980, 1991, 2000 e 2010 [página da Internet]. São Paulo: Prefeitura de São Paulo; 2012. [texto da Internet]. [acessado 2011 jan 10]. Disponível em: http://infocidade.prefeitura.sp.gov.br/htmls/7 populacao_recenseada_projetadataxas_de_c_1950 _638.html.
13. Brasil. Resolução CFN no 380 de 28 de dezembro de 2005. Dispõe sobre a definição das áreas de atuação do nutricionista e suas atribuições, estabelecer parâmetros numéricos de referência, por área de atuação e dá outras providências. Conselho Federal dos Nutricionistas 2005; 28 dez.

14. Brasil. Portaria no 648 de 28 de março de 2006. Aprova a Política Nacional de Atenção Básica, estabelecendo a revisão de diretrizes e normas para a organização da Atenção Básica para o Programa Saúde da Família (PSF) e o Programa Agentes Comunitários de Saúde (PACS). Diário Oficial da União 2006; 28 mar.

15. Brasil. Cadastro Nacional de Estabelecimentos de Saúde [página da Internet]. Brasil: Ministério da Saúde; 2008 [acessado em 2010 mar 3]. Disponível em: http://cnes.datasus.gov.br/.

16. São Paulo. Índice Paulista de Vulnerabilidade Social (IPVS). Prefeitura de São Paulo [página da Internet] 2000. [acessado 2011 jan 20]. Disponível em: http://www9.prefeitura.sp.gov.br/sempla/mm/ index.php?texto $=$ corpo\&tema_cod $=7$.

17. Bousquat A, Viana ALA, Alves D, Porto Alves MCGP, Elias PE, Oliveira VE, Manerich A, Gomes APA, Rezende AS, Lopes EZ, Cavicchioli Neto V. Avaliação do PSF pela população residente em áreas metropolitanas através de instrumento de coleta de dados informatizado [relatório final]. São Paulo: Centro de Estudos de Cultura Contemporânea (CEDEC); 2007.

18. Viana EMN, Almeida PC, Soares NT, Luna MFG, Esmeraldo GROV Adequação dos profissionais de saúde ao Programa de Saúde da Família: propostas e conquistas. Rev APS 2010; 13(1):109-117.

19. Pinto NRS, Spedo SM, Tanaka OY. (Im)possibilidades de implantar uma direção única no SUS em município de grande porte: o caso de São Paulo, Brasil. Saúde Soc 2010; 19(3):518-532.

20. Gambardella AMD, Ferreira CF, Frutuoso MFP. Situação profissional de egressos de um curso de nutrição. Rev Nutr 2000; 13(1):37-40.

21. Pádua JG de, Boog MCF. Avaliação da inserção do nutricionista na Rede Básica de Saúde dos municípios da Região Metropolitana de Campinas. Rev Nutr 2006; 19(4):413-424.

22. Santos RCL, Diez-Garcia RW. Dimensionamento de recursos humanos em serviços de alimentação e nutrição de hospitais públicos e privados. Rev Adm Pública 2011; 45(6):1805-1820.

23. Pereira DA, Vieira VL, Fiore EG, Mancuso AMC. Insegurança alimentar em região de alta vulnerabilidade social na cidade de São Paulo. SAN 2006; 13(2):34-42.

24. Vieira VL, Souza JMP, Cervato-Mancuso AM. Insegurança alimentar, vínculo mãe-filho e desnutrição infantil em área de alta vulnerabilidade social. Rev Bras Saúde Mater Infant 2010; 10(2):199-207.

25. Mattos PF, Neves AS. A Importância da Atuação do Nutricionista na Atenção Básica à Saúde. Rev Práxi. 2009; 1(2):11-15 
26. Brasil. Resolução no 5 de 07 de novembro de 2001. Institui diretrizes curriculares nacionais do curso de graduação em nutrição. Diário Oficial da União 2001; 07 nov.

27. Fernandez PMF, Voci SM, Kamata LH, Najas MS, Souza ALM. Programa Saúde da Família e as ações em nutrição em um distrito de saúde do município de São Paulo. Cien Saude Colet 2005; 10(3):749-755.

28. Maia TDM. A inserção do nutricionista na atenção primárial : o caso da microrregião de saúde de Viçosa - MG [dissertação]. São Paulo: Universidade de São Paulo; 2010.

29. Brasil. Ministério da Saúde (MS). Matriz de ações de alimentação e nutrição na atenção básica de saúde. Brasília: MS; 2009.

30. Campos GWS, Domitti AC. Apoio matricial e equipe de referência: uma metodologia para gestão do trabalho interdisciplinar em saúde. Cad Saude Publica 2007; 23(2):399-407.

Artigo apresentado em 22/07/2011

Aprovado em 31/07/2011

Versão final apresentada em 13/11/2012 